\title{
A RELAÇÃO ENTRE ESTRATÉGIAS DE APRENDIZAGEM E RENDIMENTO ESCOLAR NO ENSINO MÉDIO
}

\author{
Mônica Dias Palitot ${ }^{1}$ \\ Joseane da Silva Meireles ${ }^{1}$ \\ Francisco de Assis Toscano de Brito ${ }^{1}$ \\ Henrique Miguel de Lima Silva ${ }^{1}$ \\ Amanda Trajano Batista ${ }^{1}$
}

\section{RESUMO}

Introdução: Em contextos educacionais, os aprendentes utilizam procedimentos para facilitar a aquisição, armazenamento e posterior aplicação dos conhecimentos aprendidos, que são as estratégias que servem como recursos para uma aprendizagem bem sucedida. Dessa forma, este estudo teve por objetivo avaliar o uso de estratégias de aprendizagem e o rendimento escolar dos estudantes do Ensino Médio de escolas públicas e privadas da cidade de João Pessoa, de modo a verificar o repertório de estratégias de aprendizagem utilizado pelos mesmos. Método: A amostra foi composta por 600 estudantes, com uma faixa etária de 14 a 29 anos. Os instrumentos utilizados foram uma Escala de Avaliação das Estratégias de Aprendizagem contendo 41 itens que foi aplicada coletivamente e analisadas pelo Software SPSS. Resultados: Os resultados demonstraram que as estratégias utilizadas e que contribuem para o desenvolvimento da aprendizagem foram: fazer esquemas usando as ideias principais do texto(27\%) e criar perguntas e respostas sobre o assunto(27\%). No tocante a repetência, observou-se que $27,5 \%$ da amostra já a apresentou por pelo menos uma vez, com relação ao sexo, houve uma maior prevalência do sexo feminino $(58 \%, n=348)$ corroborando com estudos na área. Conclusão: Espera-se que os resultados desta pesquisa possam auxiliar na construção do saber prático, a fim de mobilizar novas ações na práxis pedagógica e psicopedagógica, sobretudo na elaboração de intervenções quanto ao uso de estratégias de aprendizagem.

Palavras chave: estudantes, estratégias de aprendizagem, rendimento escolar.

\section{Introdução}

O processo de ensino-aprendizagem é hoje entendido como uma construção que envolve um papel ativo por parte do aluno. Nesta perspectiva, torna-se imprescindível

\footnotetext{
${ }^{1}$ Universidade Federal da Paraíba - UFPB.
} 
que o aluno desenvolva a capacidade de estabelecer as próprias metas, planejar e monitorar seus esforços na direção de um melhor desempenho acadêmico, direcionando em certa medida, sua aprendizagem no contexto escolar (SOUZA, 2010).

As estratégias de aprendizagem são procedimentos escolhidos e coordenados pelo aluno, em função dos objetivos de saber que ele busca (PERRAUDEAU, 2009). Referem-se a recursos utilizados pelos alunos visando à assimilação, ao armazenamento e à posterior recuperação da informação, tratando-se de atividade realizada com direção e com propósito (OLIVEIRA, BORUCHOVITCH, SANTOS, 2011).

De modo geral, uma estratégia de aprendizagem envolve diversos recursos utilizados pelos estudantes ao aprender um novo conteúdo, ou desenvolver determinadas habilidades, podendo ser abrangente e generalizável à aprendizagem de várias tarefas e conteúdos ou restrita a uma tarefa específica (SOUZA, 2010). As estratégias de aprendizagem funcionam como reforçadoras da aprendizagem visto que instrumentalizam o aluno a diversificar as formas de estudo, promovendo atitudes de autoavaliação e melhora do desempenho escolar (OLIVEIRA, BORUCHOVITCH, SANTOS, 2009).

Existem diversas classificações e diferentes tipos de estratégias de aprendizagem. Todavia, a literatura vem empregando o termo estratégia de aprendizagem para designar tanto as estratégias de aprendizagem cognitivas quanto as estratégias metacognitivas (CRUVINEL, BORUCHOVITCH, 2004).

Segundo Lins, Araujo, Minervino (apud Dembo, 1994), as estratégias cognitivas referem-se a comportamentos e pensamentos que influenciam o processo de aprendizagem de maneira que a informação possa ser armazenada de forma mais eficiente, enquanto que as estratégias metacognitivas são procedimentos que o indivíduo usa para planejar, monitorar e regular o seu próprio pensamento.

As estratégias cognitivas referem-se à ação do sujeito em uma determinada tarefa; elas têm uma função executiva e para isso o sujeito se utiliza de atividades ou técnicas. Essas estratégias têm uma relação íntima com sete processos de aprendizagem: atenção, aquisição, personalização, recuperação, transferência e avaliação. Já as estratégias metacognitivas são responsáveis pela regulação dos processos mentais diante de uma tarefa de aprendizagem e favorecem a consciência da execução da ação cognitiva. Elas teriam um papel fundamental na escolha das estratégias cognitivas a serem utilizadas e 
apontam o momento e o modo de utilizá-las adequadamente. Dessa forma, podemos dizer que são elas que controlam as ações estratégicas do sujeito (FERREIRA, 2007).

$\mathrm{O}$ uso de estratégias de aprendizagem, além de instrumentalizar o aluno para o momento do estudo, também permite, por meio das estratégias metacognitivas, que este planeje, monitore e regule o seu estudo para que o aprendizado seja potencializado (OLIVEIRA, BORUCHOVITCH, SANTOS 2009). De acordo com os estudiosos do tema, parece não ser suficiente dispor de estratégias adequadas. É necessário saber como, quando e por que utilizá-las, bem como controlar sua maior ou menor eficácia e modificá-las em função da tarefa (SANTOS, BORUCHOVITCH,).

Ser estratégico é muito mais do que utilizar técnicas e métodos para aprender. O estudante que emprega estratégias é um construtor à medida que tem um papel ativo e de mediação social do seu conhecimento (OLIVEIRA, BORUCHOVITCH, SANTOS 2009). Desse modo, conhecer estratégias que facilitam o armazenamento e a utilização dos novos conhecimentos é um desafio que pode se reverter, por um lado, na remediação das dificuldades de estudo e aprendizagem. Por outro lado, implicam a melhora do desempenho acadêmico nas diferentes séries formais de ensino (OLIVEIRA, BORUCHOVITCH, SANTOS 2009).

Nota-se que embora muitos alunos conheçam um repertório de estratégias, nem sempre relatam utilizá-las com frequência. Assume-se, portanto, que esta atividade requer esforço e é marcadamente afetada pela motivação do aluno (SOUZA, 2010). Há a possibilidade de haver diferenças no uso das estratégias de aprendizagem quando se considera alunos dos sexos feminino e masculino. Verifica-se, também, que alunos mais jovens e de séries iniciais do ensino fundamental se diferenciam quanto ao uso das estratégias de aprendizagem em relação aos alunos mais velhos e de séries mais avançadas (OLIVEIRA, BORUCHOVITCH, SANTOS, 2011).

Considerando a importância das estratégias de aprendizagem para o bom desempenho acadêmico, aponta-se que, no Brasil, poucos são os instrumentos válidos e padronizados destinados a mensurar as estratégias de aprendizagem de estudantes brasileiros (OLIVEIRA, BORUCHOVTCH, SANTOS, 2009).

De acordo com Holt (1982) citado por Boruchovitch (1999), para ser um aluno de bom rendimento escolar é preciso, entre outras coisas, que se tenha consciência dos seus próprios processos mentais e do seu próprio grau de compreensão. Um aluno com 
desempenho escolar satisfatório, além de ser mais eficaz no uso e na seleção de estratégias de aprendizagem, é sempre capaz de dizer que não entendeu algo, pois ele está constantemente monitorando a sua compreensão.

De acordo com Schunk (1990) citado por Senos (1997), os alunos que se sentem seguros das suas capacidades de aprendizagem e possuem um sentimento geral de competência, exibem comportamentos de interesse e motivação para as tarefas escolares. Este comportamento permite-lhes obter um bom desempenho acadêmico, fato que contribui para validar seu sentimento pessoal de competência acadêmica e manter valores elevados de autoestima. Por outro lado, um aluno com resultados escolares negativos tenderia a sofrer o processo inverso, a menos que pudesse mobilizar formas de desvalorização desta informação negativa associada ao resultado escolar, para manter a autoestima em valores estáveis e aceitáveis.

De uma forma geral, observa-se que o rendimento dos alunos tem um escore final que pode ser representado de diversas maneiras. (OLIVEIRA, BORUCHOVTCH, SANTOS, 2009). Pode-se aventar que não somente o desempenho acadêmico poderia servir como critério para um melhor planejamento de estudo como também as estratégias de aprendizagem utilizadas no momento do estudo poderiam ser levantadas a fim de se poder orientar o aluno quanto ao seu emprego e diversificação no momento do estudo (OLIVEIRA, BORUCHOVTCH, SANTOS, 2009).

Portanto, o objetivo desse projeto é avaliar o uso de estratégias de aprendizagem e o rendimento escolar dos estudantes do Ensino Médio das escolas públicas e privadas da cidade de João Pessoa. Bem como verificar o repertório de estratégias de aprendizagem utilizado pela amostra; conhecer o perfil sociodemográfico da amostra; analisar o índice de reprovação entre os alunos que afirmam usar estratégias de aprendizagem e os que fazem menos uso destas; observar as diferenças entre meninas e meninos no que concerne a utilização das estratégias de aprendizagem para a aquisição da aprendizagem.

RPI Revista de Pesquisa Interdisciplinar, Cajazeiras, v. 2, n. 2, 128-138, jun/dez. de 2017. 


\section{Metodologia}

\section{Participantes}

Fizeram parte desse estudo 600 alunos matriculados no ensino médio na cidade de João Pessoa, sendo 300 de escolas públicas e 300 de escolas privadas.

\section{Instrumentos}

Foram utilizados, nesse estudo, os seguintes instrumentos: um questionário sociodemográfico para obter mais informações a respeito da vida familiar e acadêmica dos participantes e a Escala de Avaliação de Estratégias de Aprendizagem.

A Escala de Avaliação das Estratégias de Aprendizagem foi desenvolvida e validada por Boruchovitch e Santos (2004), com a finalidade de avaliar o repertório de estratégias de aprendizagens cognitivas e metacognitivas de alunos do ensino médio. A escala é composta por 40 itens fechados e uma questão aberta com o objetivo de investigar o uso de estratégias não mencionadas nas questões fechadas.

\section{Procedimentos}

A escolha das escolas em que seriam aplicados os instrumentos aconteceu de forma aleatória. Inicialmente, foi realizado um contato com a direção de algumas escolas públicas e privadas de João Pessoa, apresentando os objetivos do projeto e entregando a carta de anuência assinada pela orientadora do mesmo. Após as autorizações, foi dado início as aplicações dos testes que aconteceram no próprio ambiente escolar, nos horários da manhã e da tarde, de acordo com a disponibilidade das escolas. Nos dias previamente agendados, as pesquisadoras compareceram às instituições e a aplicação do caderno contendo um questionário sociodemográfico e a Escala de Avaliação das Estratégias de Aprendizagem realizou-se de forma coletiva com tempo de aproximadamente 20 minutos. Aos participantes, foi assegurada a total confidencialidade de suas respostas e esclarecido que o mesmo não comprometeria de forma alguma o seu desempenho escolar. 


\section{Resultados e discussões}

Os resultados foram subdivididos considerando-se os objetivos. Incialmente foi realizada a caracterização dos sujeitos quanto às variáveis demográficas, tais como sexo, idade, reprovação e moradia. Posteriormente, foram explorados os dados referentes ao uso de estratégias de aprendizagem na população estudada.

Quanto a variável sexo percebeu-se uma prevalência maior do sexo feminino $(58 \% n=348)$ em relação ao masculino $(42 \% n=252)$. Tal dado corrobora com estudos anteriores (Palitot e Coutinho, 2011) constatando-se também uma predominância do gênero feminino nas salas de aula. As autoras também apontam que dados nacionais da PNAD (Pesquisa Nacional por Amostra de Domicílios) indicam uma proporção maior de mulheres do que de homens frequentando o ensino médio. No perfil da amostra é possível observar que houve uma participação maior entre os estudantes do sexo feminino $(57,7 \%)$, conforme observa-se na Tabela 1 abaixo.

Tabela 1- sexo dos indivíduos.

\begin{tabular}{c|c|c}
\hline Sexo & Frequência & $\%$ \\
\hline Masculino & 252 & 42,0 \\
Feminino & 348 & $\mathbf{5 8 , 0}$ \\
Total & 600 & 100 \\
\hline
\end{tabular}

Fonte: Pesquisa Direta 2011/2012

Quanto à história escolar, $(72,5 \%, n=435)$ dos alunos nunca foram reprovados e $(27,5 \%, n=165)$ da amostra já haviam sido reprovados pelo menos uma vez (Tabela 2$)$.

Tabela 2- Reprovação dos indivíduos.

\begin{tabular}{c|c|c}
\hline Reprovação & Frequência & $\%$ \\
\hline Sim & 165 & 27,5 \\
Não & 435 & $\mathbf{7 2 , 5}$ \\
Total & 600 & 100,0 \\
\hline
\end{tabular}

Fonte: Pesquisa Direta 2011/2012

A idade máxima dos participantes foi de 29 anos e a mínima de 14, conforme observa-se na Tabela 3 abaixo. 
Tabela 3- Idade dos indivíduos da amostra.

\begin{tabular}{c|c|c}
\hline Idade & Frequência & $\%$ \\
\hline 13 & 4 &, 7 \\
14 & 57 & 9,5 \\
15 & 140 & 23,3 \\
16 & 170 & 28,3 \\
17 & 141 & 23,5 \\
18 & 61 & $\mathbf{1 0 , 2}$ \\
19 & 14 & 2,3 \\
20 & 8 & 1,3 \\
21 & 1 &, 2 \\
22 & 2 &, 3 \\
24 & 1 &, 2 \\
29 & 1 &, 2 \\
Total & 600 & 100,0 \\
\hline
\end{tabular}

Fonte: Pesquisa Direta 2011/2012

Dos participantes, $(86,3 \% n=518)$ moram com os pais, $(7,8 \% n=47)$ moram com os avós e $(5,8 \% n=35)$ moram com outros, conforme pode-se verificar na Tabela 4.

Tabela 4- Família com quem os indivíduos moram.

\begin{tabular}{c|c|c}
\hline Família & Frequência & $\%$ \\
\hline Pais & 518 & $\mathbf{8 6 , 3}$ \\
Avós & 47 & $\mathbf{7 , 8}$ \\
Outros & 35 & $\mathbf{5 , 8}$ \\
Total & 600 & $\mathbf{1 0 0 , 0}$ \\
\hline
\end{tabular}

Fonte: Pesquisa Direta 2011/2012

No que concerne ao uso das estratégia de aprendizagem, foi realizado uma análise para verificar a frequência com que os participantes utilizavam tais estratégias, se "sempre", "algumas vezes" ou "nunca". Desta forma, pode-se apreender que a estratégia sempre utilizada foi "quando lê um texto o reescreve com suas palavras"(27\%), já aquela utilizada algumas vezes corresponde a "ao ler o texto repete em voz alta"(61,2\%), e a estratégia que foi relatada como nunca realizada "percebe quando não entende o que esta lendo"(67,2\%). Os dados também demonstraram que 
80,3\% dos estudantes citaram o fato de perceber quando tem dificuldades para aprender algum assunto.

Outra estratégia muito citada refere-se à compreensão da leitura (item 26), na qual 74\% dos participantes afirmam que costumam perceber quando não entendem a leitura sendo que 70,5\% param para ler novamente (item 27). A maioria das estratégias foi escolhida pelos participantes como sendo utilizadas "algumas vezes".

Verificou-se que, 30,7\% dos participantes admitiram que costumam fazer o dever de casa na última hora (item 28), enquanto que $23,2 \%$ costumam esquecer de fazer o dever de casa (item 38) e 21,3\% conseguem ir até o final da tarefa mesmo ela sendo difícil ou chata (item 8). 27,8\% costumam se distrair enquanto estudam (item 37) e $16,7 \%$ costumam se sentir nervosos quando fazem as tarefas e com medo acabam errando (item 39).

Como afirma Sukiennik et al.(2000) é preciso ficar atento a alguns sinais de alerta emitidos pelo adolescente como a piora progressiva em seu desempenho escolar, tanto pela falta de motivação como também por uma diminuição transitória de sua capacidade cognitiva. A realização de tarefas em casa são estratégias de suma importância para o processo de aprendizagem uma vez que reforçaram a aprendizagem iniciada em sala de aula, e/ou possibilitará ao estudante perceber as suas dúvidas.

Embora a maior parte das estratégias de aprendizagem tenha sido citada como sendo utilizadas "algumas vezes", outras mencionadas como nunca utilizadas merecem ser destacadas; $31,7 \%$ dos participantes quando leem um texto, não escrevem com suas palavras o que entenderam (item7); 34,7\% não criam perguntas e respostas sobre o assunto estudado (item 12), 47,2\% não costumam fazer esquemas (item 16), 38,0\% não procuram no dicionário o significado das palavras (item 22), 38,2\% não costumam ler outros textos e livros sobre o assunto estudado (item 25) e 56,2\% dos participantes não utilizam outros tipos de estratégias diferente das citadas (item 41).

A não realização de estratégias de aprendizagem como as citadas acima demonstram que ocorrerá uma lacuna no uso destas, pois se sabe que o uso de estratégias tais como: escrever com as próprias palavras o que foi entendido, ou criar perguntas sobre o assunto estudado e o hábito quanto ao uso do dicionário poderiam facilitar a absorção, a compreensão dos conteúdos ministrados em sala de aula. 
No que concerne ao rendimento escolar dos alunos, pode-se constatar que a maioria dos participantes $(30,0 \% n=180)$ alegou apresentar melhor rendimento em Português, $(16,2 \% n=97)$ em Matemática, (5,5\% n=33) em Física, (5,5\%n=33) em Química, $(13,7 \% n=82)$ em Biologia, $(4,8 \% n=29)$ em Língua estrangeira, $(6,8 \% n=41) \mathrm{em}$ Geografia, $(11,8 \%$ n=71) em História e (5,7\% n=34) em Educação Física.

Os resultados demonstraram que, de um modo geral, a maioria dos participantes fazem uso de estratégias cognitivas e metacognitivas no momento de estudo. Neste sentido, Boruchovitch (1999), Mckeachie e cols. (1985) e Reay (2006) citados por Oliveira, Boruchovitch e Santos (2009), enfatizam que o uso das estratégias de aprendizagem facilita a aprendizagem, uma vez que viabiliza a aquisição e a posterior recuperação e uso da informação, bem como fomenta o bom desempenho acadêmico.

Em relação às estratégias mais utilizadas, os resultados apontam que os estudantes costumam perceber quando não estão compreendendo a leitura, porém, alguns não param para ler novamente. Nesta perspectiva, Lins, Araujo e Minervino (2011) concluem que, os estudantes, ao adotarem determinadas rotinas, não conseguem desvencilhar-se delas porque é difícil modificar hábitos enraizados e, para fazê-lo, é preciso que novos comportamentos sejam aprendidos através do ensino sistemático.

Souza (2010) conclui que as estratégias cognitivas estão diretamente relacionadas à execução de tarefas, ao passo que as estratégias metacognitivas geralmente são mais amplas e implicam na organização, regulação e mesmo na avaliação do uso das estratégias cognitivas. Ressalta-se ainda que o monitoramento pode informar se uma estratégia em particular está promovendo resultados efetivos e se o estudante está alcançando níveis de desempenho adequados aos seus padrões internos. Assim, pode-se afirmar que as estratégias permitem planejar e monitorar o próprio desempenho.

\section{Conclusão}

Para Almeida (2002) citado por Oliveira, Boruchovitch e Santos (2009), a aprendizagem é um processo ativo que deve ser fomentado por meio de métodos que permitam ao aluno o uso da iniciativa, primando, inclusive, pelo senso de responsabilidade do aluno. Pode-se então concluir que, os alunos que utilizam de 
estratégias de aprendizagem são também aqueles que apresentam um bom rendimento escolar.

Foi visto que, alguns alunos se esquecem de fazer os deveres de casa ou fazem de última hora, não fazem esquemas e não procuram o significado de palavras desconhecidas no dicionário. Diante disso, Boruchovtch (1999) ressalta que, as estratégias de aprendizagem podem ser ensinadas para os alunos que apresentem um baixo rendimento escolar. Segundo a autora, é possível ensinar a todos os alunos a expandir notas de aulas, a sublinhar pontos importantes de um texto, a monitorar a compreensão na hora da leitura, usar técnicas de memorização, fazer resumos, entre outras estratégias. Isso pode tornar-se uma solução para que esses alunos além de melhorar seu rendimento possam sentir-se motivados.

Espera-se que os resultados desta pesquisa possam auxiliar na construção do saber prático, a fim de mobilizar novas ações na práxis pedagógica e psicopedagógica, sobretudo na elaboração de intervenções quanto ao uso de estratégias de aprendizagem tanto no ambiente escolar quanto em casa, visando à melhoria da aprendizagem nos alunos do ensino médio brasileiro.

\section{Referências}

BORUCHOVITCH, E. Estratégias de aprendizagem e desempenho escolar: considerações para a prática educacional. Psicologia: Reflexão e Crítica, Porto Alegre, v.12, n.2, p.361-376, 1999.

CRUVINEL, M.; BORUCHOVITCH, E. Sintomas depressivos, estratégias de aprendizagem e rendimento escolar de alunos do ensino fundamental. Psicologia em estudo, Maringá, v.9, n.3, p.369-378, 2004.

\section{FERREIRA, L.F. Estratégias de aprendizagem do aluno de $5^{\mathbf{a}}$ série na} resolução de situação-problema. Paraná, 2007.

LINS, M.R.C.; ARAÚJO, M.R; MINERVINO, C.A.S.M. Estratégias de aprendizagem empregadas por estudantes do Ensino Fundamental. Revista Semestral da Associação Brasileira de Psicologia Escolar e Educacional, São Paulo, v.15, n.1, p.63-70, 2011.

OLIVEIRA, K.L.; BORUCHOVITCH, E.; SANTOS, A.A.A. Estratégias de aprendizagem no ensino fundamental: análise por gênero, série escolar e idade. Psico, Porto Alegre, v.42, n.1, p.98-105, 2011. 
PALITOT, M.D., COUTINHO, M.P.L. Uso de Estratégias de Aprendizagem no Ensino Médio da Região Nordeste do Brasil. IN: Métodos de pesquisa em psicologia social: perspectivas qualitativas e quantitativas. ED. Universitária: João Pessoa, pp.433460, 2011.

PERRAUDEAU, M. Estratégias de aprendizagem: como acompanhar os alunos na aquisição dos saberes. Ed. Artmed, Porto Alegre, 2009.

SENOS, J. Identidade social, auto-estima e resultados escolares. Análise Psicológica, v.1, p.123-197, 1997.

SOUZA, L.F.N.I. Estratégias de aprendizagem e fatores motivacionais relacionados. Educar, Curitiba, n.36, p.95-107, 2010. 